\title{
Liouville property, Wiener's test and unavoidable sets for Hunt processes
}

\author{
Wolfhard Hansen
}

\begin{abstract}
Let $(X, \mathcal{W})$ be a balayage space, $1 \in \mathcal{W}$, or - equivalently - let $\mathcal{W}$ be the set of excessive functions of a Hunt process on a locally compact space $X$ with countable base such that $\mathcal{W}$ separates points, every function in $\mathcal{W}$ is the supremum of its continuous minorants and there exist strictly positive continuous $u, v \in \mathcal{W}$ such that $u / v \rightarrow 0$ at infinity. We suppose that there is a Green function $G>0$ for $X$, a metric $\rho$ for $X$ and a decreasing function $g:[0, \infty) \rightarrow(0, \infty]$ having the doubling property such that $G \approx g \circ \rho$.

Assuming that the constant function 1 is harmonic and balls are relatively compact, is is shown that every positive harmonic function is constant (Liouville property) and that Wiener's test at infinity shows, if a given set $A$ in $X$ is unavoidable, that is, if the process hits $A$ with probability one, wherever it starts.

An application yields that locally finite unions of pairwise disjoint balls $B\left(z, r_{z}\right), z \in Z$, which have a certain separation property with respect to a suitable measure $\lambda$ on $X$ are unavoidable if and only if, for some/any point $x_{0} \in X$, the series $\sum_{z \in Z} g\left(\rho\left(x_{0}, z\right)\right) / g\left(r_{z}\right)$ diverges.

The results generalize and, exploiting a zero-one law for hitting probabilities, simplify recent work by S. Gardiner and M. Ghergu, A. Mimica and Z. Vondraček, and the author.

Keywords: Hunt process; balayage space; unavoidable set; zero-one law; Green function; equilibrium measure; capacity; doubling property; Liouville property; Wiener's criterion; Lévy process.
\end{abstract}

MSC: 31B15, 31C15, 31D05, 60J25, 60J45, 60J65, 60J75.

\section{Preliminaries and main results}

Let $X$ be a locally compact space with countable base. Let $\mathcal{C}(X)$ denote the set of all continuous real functions on $X$ and let $\mathcal{B}(X)$ be the set of all Borel measurable numerical functions on $X$. The set of all (positive) Radon measures on $X$ will be denoted by $\mathcal{M}(X)$.

Moreover, let $\mathcal{W}$ be a convex cone of positive lower semicontinuous numerical functions on $X$ such that $1 \in \mathcal{W}$ and $(X, \mathcal{W})$ is a balayage space (see [2], 6] or [11, Appendix]). In particular, the following holds: 
(C) $\mathcal{W}$ separates the points in $X$,

$$
w=\sup \{v \in \mathcal{W} \cap \mathcal{C}(X): v \leq w\} \quad \text { for every } w \in \mathcal{W},
$$

and there are strictly positive $u, v \in \mathcal{W} \cap \mathcal{C}(X)$ such that $u / v \rightarrow 0$ at infinity.

Then there exists a Hunt process $\mathfrak{X}$ on $X$ such that $\mathcal{W}$ is the set $E_{\mathbb{P}}$ of excessive functions for the transition semigroup $\mathbb{P}=\left(P_{t}\right)_{t>0}$ of $\mathfrak{X}$ (see [2, IV.7.6] or [11, Appendix]), that is,

$$
\mathcal{W}=\left\{v \in \mathcal{B}^{+}(X): \sup _{t>0} P_{t} v=v\right\}
$$

We note that, conversely, given any sub-Markov semigroup $\mathbb{P}=\left(P_{t}\right)_{t>0}$ on $X$ such that $(\mathrm{C})$ is satisfied by its convex cone $E_{\mathbb{P}}$ of excessive functions, $\left(X, E_{\mathbb{P}}\right)$ is a balayage space, and $\mathbb{P}$ is the transition semigroup of a Hunt process (see [6, Corollary 2.3.8] or [11, Corollary A.5]).

For every numerical function $f$ on $X$, let

$$
R_{f}:=\inf \{v \in \mathcal{W}: v \geq f\} .
$$

In particular, for every subset $A$ of $X$, we have reduced functions $R_{u}^{A}, u \in \mathcal{W}$, and reduced measures $\varepsilon_{x}^{A}, x \in X$, defined by

$$
R_{u}^{A}:=R_{1_{A} u}=\inf \{v \in \mathcal{W}: v \geq u \text { on } A\} \quad \text { and } \quad \int u d \varepsilon_{x}^{A}=R_{u}^{A}(x)
$$

Clearly, $R_{u}^{A} \leq u$ on $X$ and $R_{u}^{A}=u$ on $A$. If $A$ is open, then

$$
R_{u}^{A} \in \mathcal{W}
$$

For a general subset $A$, the greatest lower semicontinuous minorant $\hat{R}_{1}^{A}$ of $R_{1}^{A}$ is contained in $\mathcal{W}$, and $\hat{R}_{1}^{A}=R_{1}^{A}$ on $A^{c}$ (see [2, p. 243]).

If $A$ is Borel measurable, then, for every $x \in X$,

$$
R_{1}^{A}(x)=P^{x}\left[T_{A}<\infty\right],
$$

where $T_{A}(\omega):=\inf \left\{t \geq 0: X_{t}(\omega) \in A\right\}$ and, more generally,

$$
\varepsilon_{x}^{A}(B)=P^{x}\left[X_{T_{A}} \in B ; T_{A}<\infty\right]
$$

for every Borel measurable set $B$ in $X$ (see [2, VI.3.14]).

For every open set $U$ in $X$, let $\mathcal{H}^{+}(U)$ denote the set of all functions $h \in \mathcal{B}^{+}(X)$ which are harmonic on $U$ (in the sense of [2]), that is, such that $\left.h\right|_{U} \in \mathcal{C}(U)$ and

$$
\varepsilon_{x}^{V^{c}}(h):=\int h d \varepsilon_{x}^{V^{c}}=h(x)
$$

for every open $V$ such that $x \in V$ and $\bar{V}$ is a compact in $U$. Let $\tilde{\mathcal{H}}^{+}(U)$ denote the (possibly larger) set of all $h \in \mathcal{B}^{+}(X)$ such that (1.3) holds, whenever $V$ is open, $x \in V$ and $\bar{V}$ is compact in $U$. By [2, VI.2.6]), for every set $A$ in $X$,

$$
R_{u}^{A} \in \mathcal{H}^{+}(X \backslash \bar{A}), \quad \text { if } u \in \mathcal{W}, u \leq w \in \mathcal{W} \cap \mathcal{C}(X) .
$$


In the following let us assume that the constant function 1 is harmonic on $X$.

We recall that a subset $A$ of $X$ is called unavoidable, if $R_{1}^{A}=1$ (or, equivalently, $\left.\hat{R}_{1}^{A}=1\right)$. Otherwise, it is called avoidable, that is, $A$ is avoidable, if there exists $x \in X$ such that $R_{1}^{A}(x)<1$. The following zero-one law will play an important role (for its proof and the proof of the subsequent corollary see [11, Proposition 2.3]).

PROPOSITION 1.1. For every $A \subset X$,

$$
R_{1}^{A}=1 \quad \text { or } \quad \inf _{x \in X} R_{1}^{A}(x)=0 .
$$

COROLLARY 1.2. If $A \subset X$ is unavoidable and $B \subset X, \gamma>0$ such that $R_{1}^{B} \geq \gamma$ on $A$, then $B$ is unavoidable.

Moreover, we recall the following elementary fact (see [11, Lemma 2.2]).

PROPOSITION 1.3. Let $A$ be an unavoidable set in $X$ and let $\left(B_{n}\right)$ be a sequence of relatively compact sets in $X 1$ Then the following hold.

(a) For every $n \in \mathbb{N}$, the set $A \backslash\left(B_{1} \cup B_{2} \cup \cdots \cup B_{n}\right)$ is unavoidable.

(b) If $A \subset \bigcup_{n \in \mathbb{N}} B_{n}$, then $\sum_{n \in \mathbb{N}} R_{1}^{B_{n}}=\infty$.

By definition, a potential on $X$ is a function $p \in \mathcal{W}$ such that, for every relatively compact open set $U$ in $X$, the function $R_{p}^{X \backslash U}$ is continuous and real on $U$ and

$$
\inf \left\{R_{p}^{X \backslash U}: U \text { relatively compact open in } X\right\}=0 .
$$

By [6, Proposition 4.2.10], a function $p \in \mathcal{W} \cap \mathcal{C}(X)$ is a potential if and only if there exists a strictly positive $q \in \mathcal{W} \cap \mathcal{C}(X)$ such that $p / q$ vanishes at infinity. Let $\mathcal{P}$ denote the set of all continuous real potentials on $X$.

In the following let us assume that there is a Green function $G$ for $(X, \mathcal{W})$ which is related to a metric for the topology of $X$ :

ASSUMPTION 1.4. We have a Borel measurable function $G: X \times X \rightarrow(0, \infty]$ and a metric $\rho$ for $X$ such that the following hold:

(i) For every $y \in X, G(\cdot, y)$ is a potential which is harmonic on $X \backslash\{y\}$.

(ii) For every potential $p$ on $X$, there exists a measure $\mu$ on $X$ such that

$$
p=G \mu:=\int G(\cdot, y) d \mu(y) .
$$

(iii) There exist a decreasing function $g:[0, \infty) \rightarrow(0, \infty]$ and a constant $c \geq 1$ such that

$$
c^{-1} g \circ \rho \leq G \leq c g \circ \rho .
$$

REMARKS 1.5. 1. Having (i), each of the following properties implies (ii).

\footnotetext{
${ }^{1}$ It is easily seen that it is sufficient to assume that the the functions $R_{1}^{B_{n}}, n \in \mathbb{N}$, are $\mathcal{P}$-bounded.
} 
- $G$ is lower semicontinuous on $X \times X$, continuous outside the diagonal, the potential kernel $V_{0}:=\int_{0}^{\infty} P_{t} d t$ of $\mathfrak{X}$ is proper, and there is a measure $\mu$ on $X$ such that $V_{0} f=\int G(\cdot, y) f(y) d \mu(y), f \in \mathcal{B}^{+}(X)$ (see [13] and [2, III.6.6]).

- $G$ is locally bounded off the diagonal, each function $G(x, \cdot)$ is lower semicontinuous on $X$ and continuous on $X \backslash\{x\}$, and there exists a measure $\nu$ on $X$ such that $G \nu \in \mathcal{C}(X)$ and $\nu(U)>0$, for every finely open $U \neq \emptyset$ (the latter holds, for example, if $\left.V_{0}(x, \cdot) \ll \nu, x \in X\right)$. See [10, Theorem 4.1].

2. For a discussion of (iii) and the later doubling property (1.11) see the Appendix.

The measure in (1.6) is uniquely determined and, given any measure $\mu$ on $X$ such that $p:=G \mu$ is a potential, the complement of the support of $\mu$ is the largest open set, where $p$ is harmonic (see, for example, [10, Proposition 5.2 and Lemma 2.1]).

Suppose that $A$ is a subset of $X$ such that $\hat{R}_{1}^{A}$ is a potential. Then there is a unique measure $\mu_{A}$ on $X$, the equilibrium measure for $A$, such that

$$
\hat{R}_{1}^{A}=G \mu_{A}
$$

If $A$ is open, then $\hat{R}_{1}^{A}=R_{1}^{A} \in \mathcal{H}^{+}(X \backslash \bar{A})$, and hence $\mu_{A}$ is supported by $\bar{A}$. We observe that, for a general balayage space, this may already fail if $A$ is compact (see [2, V.9.1]).

We define inner capacities for open sets $U$ in $X$ by

$$
\operatorname{cap}_{*} U:=\sup \{\|\mu\|: \mu \in \mathcal{M}(X), \mu(X \backslash U)=0, G \mu \leq 1\}
$$

and outer capacities for arbitrary sets $A$ in $X$ by

$$
\operatorname{cap}^{*} A:=\inf \left\{\operatorname{cap}_{*} U: U \text { open neighborhood of } A\right\} \text {. }
$$

Of course, the function $U \mapsto \operatorname{cap}_{*} U, U$ open in $X$, is increasing. Hence the function

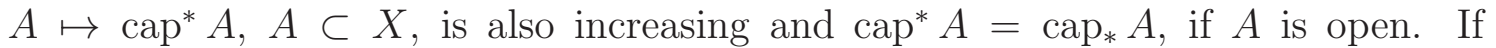
$\operatorname{cap}_{*} A=\operatorname{cap}^{*} A$, we may simply write cap $A$ and speak of the capacity of $A$.

The capacity of open sets $U$ is essentially determined by the total mass of equilibrium measures for open sets which are relatively compact in $U$ :

LEMMA 1.6. For every open set $U$ in $X$,

$$
\operatorname{cap} U \geq \sup \left\{\left\|\mu_{V}\right\|: V \text { open and } \bar{V} \text { compact in } U\right\} \geq c^{-2} \operatorname{cap} U \text {. }
$$

Proof. The first inequality is trivial. To prove the second inequality, let $\mu \in \mathcal{M}(X)$ such that $\mu(X \backslash U)=0$ and $G \mu \leq 1$, and let $K$ be a compact in $U$. We may choose an open neighborhood $V$ of $K$ such that $\bar{V}$ is compact in $U$. Then

$$
\begin{aligned}
\left\|1_{K} \mu\right\| & =\int_{K} G \mu_{V} d \mu \leq \iint G(x, y) d \mu_{V}(y) d \mu(x) \\
& \leq c^{2} \iint G(y, x) d \mu(x) d \mu_{V}(y)=c^{2} \int G \mu(y) d \mu_{V}(y) \leq c^{2}\left\|\mu_{V}\right\| .
\end{aligned}
$$


For $x \in X$ and $0<r<t$, we define balls $B(x, r)$ and shells $S(x, r, t)$ by

$$
B(x, r):=\{y \in X: \rho(x, y)<r\}, \quad S(x, r, t):=\{y \in X: r \leq \rho(x, y)<t\} .
$$

Let us immediately note some elementary properties of $R_{1}^{B(x, r)}$ and cap $B(x, r)$.

PROPOSITION 1.7. Let $x \in X, r>0$ and $B:=B(x, r)$. Then $R_{1}^{B}$ is a potential which is $\mathcal{P}$-bounded,

$$
\begin{gathered}
R_{1}^{B} \leq c \frac{G(\cdot, x)}{g(r)} \leq c^{2} \frac{g(\rho(\cdot, x))}{g(r)}, \quad\left\|\mu_{B}\right\| \vee \operatorname{cap} B \leq c g(r)^{-1}, \\
R_{1}^{B} \geq c^{-1} \operatorname{cap} B \cdot g(\rho(\cdot, x)+r) .
\end{gathered}
$$

Proof. We know that $R_{1}^{B} \in \mathcal{W}$ (see (1.1)). Moreover, $G(\cdot, x)$ is a potential and $G(\cdot, x) \geq c^{-1} g(r)$ on $B$. Hence $R_{1}^{B} \leq \min \{1, c G(\cdot, x) / g(r)\} \in \mathcal{P}$. In particular, $R_{1}^{B}$ is a potential.

Moreover, let $\mu \in \mathcal{M}(X)$ such that $\mu(X \backslash \bar{B})=0$ and $\int G(\cdot, z) d \mu(z)=G \mu \leq 1$. Since $c^{-1} g(r) \leq G(x, \cdot)$ on $\bar{B}$, we see that $c^{-1} g(r)\|\mu\| \leq 1$.

If even $\mu(X \backslash B)=0$, then, by the minimum principle (see [2, III.6.6]), $R_{1}^{B} \geq G \mu$. Let $y \in X$. For all $z \in B, \rho(z, y)<\rho(y, x)+r$, and hence $\left.G(y, z) \geq c^{-1} g(\rho(y, x)+r)\right)$. Thus $R_{1}^{B}(y) \geq G \mu(y) \geq c^{-1} g(\rho(y, x)+r)\|\mu\|$.

ASSUMPTION 1.8. From now on we assume, in addition, the following.

(iv) Doubling property: There exist $c_{D} \geq 1$ and $0 \leq R_{0}<\infty$ such that

$$
g(r / 2) \leq c_{D} g(r) \quad \text { for every } r>R_{0}
$$

(v) All balls $B(x, r), x \in X, r>0$, are relatively compact.

REMARKS 1.9. 1. We note that Assumptions 1.4 and 1.8 are satisfied by rather general isotropic Lévy processes (often with $R_{0}=0$; see [4] and [7] for details).

2. If (1.11) is known to hold with some $R_{0}>0$, then we may replace $R_{0}$ by any $R_{0}^{\prime} \in\left(0, R_{0}\right.$ ) (at the expense of taking a larger $c_{D}^{\prime}$ ), since $0<R_{0}^{\prime}<r \leq R_{0}$ implies that $g(r / 2) \leq g\left(R_{0}^{\prime} / 2\right) \leq g\left(R_{0}^{\prime} / 2\right) g\left(R_{0}\right)^{-1} g(r)$.

3. Since the function 1 is harmonic, $X$ cannot be compact, and hence (v) implies that balls are proper subsets of $X$.

Having the doubling property for $g$, there is a close relation between estimates which are reverse to the ones in (1.9).

PROPOSITION 1.10. Let $x \in X, r>R_{0}, B:=B(x, r), C \geq 1$, and $\tilde{C}:=c^{2} c_{D} C$. Then the following hold.

(a) If cap $B \geq C^{-1} g(r)^{-1}$, then $R_{1}^{B} \geq \tilde{C}^{-1} G(\cdot, x) / g(r)$ on $X \backslash B$.

(b) If $R_{1}^{B} \geq C^{-1} G(\cdot, x) / g(r)$ on $X \backslash B$, then cap $B \geq \tilde{C}^{-1} g(r)^{-1}$. 
Proof. (a) Immediate consequence of (1.10): it suffices to note that, for every $y \in B^{c}$, $\rho(y, x)+r \leq 2 \rho(y, x)$ and hence $g(\rho(y, x)+r) \geq c_{D}^{-1} g(\rho(y, x)) \geq\left(c c_{D}\right)^{-1} G(y, x)$.

(b) Let $z \in X \backslash B(x, 2 r), a:=\rho(x, z)$, and $\varepsilon>0$. Then there exists $0<r^{\prime}<r$ such that $V:=B\left(x, r^{\prime}\right)$ satisfies $R_{1}^{V}(z)+\varepsilon>R_{1}^{B}(z) \geq(c C)^{-1} g(a) / g(r)$. Moreover,

$$
R_{1}^{V}(z)=\int G(z, y) d \mu_{V}(y) \leq c g(a / 2)\left\|\mu_{V}\right\| \leq c c_{D} g(a) \operatorname{cap} B,
$$

since $a / 2 \leq a-r<\rho(z, \cdot)$ on $B$. Thus $\tilde{C}^{-1} g(r)^{-1} \leq \operatorname{cap} B$.

Our main theorems are the following.

THEOREM 1.11 (Liouville property). Every function in $\tilde{\mathcal{H}}^{+}(X)$ is constant.

THEOREM 1.12 (Wiener's test). Let $A$ be a subset of $X, x_{0} \in X, R>0, \gamma>1$. Then $A$ is unavoidable if and only if

$$
\sum_{n \in \mathbb{N}} g\left(\gamma^{n} R\right) \operatorname{cap}^{*}\left(A \cap S\left(x_{0}, \gamma^{n} R, \gamma^{n+1} R\right)\right)=\infty .
$$

For the next two corollaries we suppose, in addition, that we have a measure $\lambda \in \mathcal{M}(X)$ with $\operatorname{supp}(\lambda)=X$ and such that, for some $c_{0} \geq 1$, the normalized restrictions $\lambda_{B(x, r)}:=(\lambda(B(x, r)))^{-1} 1_{B(x, r)} \lambda$ of $\lambda$ on $B(x, r), x \in X, r>R_{0}$, satisfy

$$
G \lambda_{B(x, r)} \leq c_{0} g(r)
$$

so that, in particular,

$$
\operatorname{cap} B(x, r) \geq c_{0}^{-1} g(r)^{-1}
$$

(for many Lévy processes, the Lebesgue measure will have this property; see [7]).

COROLLARY 1.13. Let $A$ be a union of pairwise disjoint balls $B\left(z, r_{z}\right), z \in Z$, where $Z \subset X$ is locally finite and $r_{z}>4 R_{0}$, and let $x_{0} \in X \backslash Z$ such that

$$
\inf _{z, z^{\prime} \in Z, z \neq z^{\prime}} \frac{\lambda\left(B\left(z, \rho\left(z, z^{\prime}\right) / 4\right)\right)}{\lambda\left(B\left(x_{0}, 4 \rho\left(x_{0}, z\right)\right)\right)} \cdot \frac{g\left(r_{z}\right)}{g\left(\rho\left(x_{0}, z\right)\right)}>0 .
$$

Then $A$ is unavoidable if and only if $\sum_{z \in Z} g\left(\rho\left(x_{0}, z\right)\right) / g\left(r_{z}\right)=\infty$.

DEFINITION 1.14. We shall say that pairwise disjoint balls $B\left(z, r_{z}\right), z \in Z$, are regularly located if the following hold:

- There exists $\varepsilon>0$ such that $\rho\left(z, z^{\prime}\right) \geq \varepsilon$, for all $z, z^{\prime} \in Z, z \neq z^{\prime}$.

- There exists $R>0$ such that every ball of radius $R$ contains a point of $Z$.

- There exists a decreasing function $\phi:(0, \infty) \rightarrow\left(4 R_{0}, \infty\right)$ and $C>1$ such that

$$
\phi\left(\rho\left(x_{0}, z\right)\right)<r_{z}<C \phi\left(\rho\left(x_{0}, z\right)\right), \quad z \in Z .
$$

Under mild additional assumptions on $\lambda$ (see Section 5), which are satisfied if $X=\mathbb{R}^{d}, \rho$ is the Euclidean metric and $\lambda$ is Lebesgue measure, the following holds.

COROLLARY 1.15. Let $A$ be a union of balls $B\left(z, r_{z}\right), z \in Z$, in $X$ which are regularly located. Then $A$ is unavoidable if and only if $\sum_{z \in Z} g\left(\rho\left(x_{0}, z\right)\right) / g\left(r_{z}\right)=\infty$. 


\section{Liouville property}

PROPOSITION 2.1. Let $U$ be an open set in $X$ and $B:=B\left(x_{0}, R\right), x_{0} \in U$, $R>R_{0}$, such that the closure of $B^{\prime}:=B\left(x_{0}, 2 R\right)$ is contained in $U$. Then

$$
\sup h(B) \leq\left(c c_{D}\right)^{2} \inf h(B) \quad \text { for every } h \in \mathcal{W} \cap \tilde{\mathcal{H}}^{+}(U) .
$$

Proof. We may choose $\varphi_{n} \in \mathcal{K}^{+}(X), n \in \mathbb{N}$, such that $\varphi_{n} \uparrow 1_{X \backslash \overline{B^{\prime}}} h$. Then

$$
h_{n}:=R_{\varphi_{n}} \in \mathcal{P} \cap \mathcal{H}^{+}\left(X \backslash \operatorname{supp}\left(\varphi_{n}\right)\right), \quad n \in \mathbb{N},
$$

by [2, III.2.4, III.5.6]. So there exist measures $\mu_{n}$ on $X, n \in \mathbb{N}$, such that

$$
h_{n}=G \mu_{n} \quad \text { and } \quad \mu_{n}\left(B^{\prime}\right)=0 .
$$

For all $x, y \in B$, and $z \in X \backslash B^{\prime}, \rho(x, z)<R+\rho\left(x_{0}, z\right) \leq 3\left(\rho\left(x_{0}, z\right)-R\right)<3 \rho(y, z)$, hence, defining $K:=\left(c c_{D}\right)^{2}, G(x, z) \leq c g(\rho(x, z)) \leq c c_{D}^{2} g(\rho(y, z)) \leq K G(y, z)$ and

$$
h_{n}(x)=\int G(x, z) d \mu_{n}(z) \leq K \int G(y, z) d \mu_{n}(z) \leq K h_{n}(y)
$$

for every $n \in \mathbb{N}$. The sequence $\left(h_{n}\right)$ is increasing to a function $u \in \mathcal{W}$ which, by (2.2), satisfies $\sup u(B) \leq K \inf u(B)$. Clearly, $u \leq h$ on $X$ and $u=h$ on $X \backslash \bar{B}^{\prime}$.

Let $V$ be an open neighborhood of $\overline{B^{\prime}}$ such that $\bar{V}$ is compact in $U$. Then $u \geq R_{h}^{V^{c}}$, since $V^{c} \subset X \backslash \bar{B}^{\prime}$, and, for every $x \in V, u(x) \geq \varepsilon_{x}^{V^{c}}(h)=h(x)$. Thus $u=h$ completing the proof.

COROLLARY 2.2 (Liouville property). Every function in $\tilde{\mathcal{H}}^{+}(X)$ is constant.

Proof. For every relatively compact open set $V$ in $X$ and every $f \in \mathcal{B}^{+}(X)$, the function $H_{V} f$ is lower semicontinuous on $V$ (see [2, III.3.4]). Therefore $\tilde{\mathcal{H}}^{+}(X) \subset \mathcal{W}$ (see, for example, [2, II.5.5]), and we obtain that (2.1) holds for all $h \in \tilde{\mathcal{H}}^{+}(X)$ and balls $B\left(x_{0}, r\right), x_{0} \in X, r>R_{0}$.

Now the claim follows immediately by a well known standard argument. (Let $h \in \tilde{\mathcal{H}}^{+}(X)$ and $a:=\inf h(X)$. Then $h^{\prime}:=h-a \in \tilde{\mathcal{H}}^{+}(X)$. Given $\varepsilon>0$, there exists $x_{0} \in X$ such that $h^{\prime}\left(x_{0}\right)<\varepsilon$ and hence, considering $x \in X$ and $R>R_{0} \vee \rho\left(x, x_{0}\right)$, we obtain that $h^{\prime}(x) \leq\left(c c_{D}\right)^{2} h^{\prime}\left(x_{0}\right)<\left(c c_{D}\right)^{2} \varepsilon$. Thus $h^{\prime}=0$.)

\section{Proof of Wiener's test}

One direction of Wiener's test is an easy consequence of Proposition 1.3(b). We only have to use the definition of cap* and note the simple fact that, for every open set $V$ which is contained in an open ball, the reduced function $R_{1}^{V}$ is a potential, by Proposition 1.7.

PROPOSITION 3.1. Let $A$ be an unavoidable set in $X, x_{0} \in X, R>0, \gamma>1$. Then

$$
\sum_{n \in \mathbb{N}} g\left(\gamma^{n} R\right) \operatorname{cap}^{*}\left(A \cap S\left(x_{0}, \gamma^{n} R, \gamma^{n+1} R\right)\right)=\infty .
$$


Proof. For $n \in \mathbb{N}$, there are open neighborhoods $U_{n}$ of $A_{n}:=A \cap S\left(x_{0}, \gamma^{n} R, \gamma^{n+1} R\right)$ in $S\left(x_{0}, \gamma^{n} R / 2, \gamma^{n+1} R\right)$ such that

$$
\operatorname{cap} U_{n} \leq \operatorname{cap}^{*} A_{n}+2^{-n}
$$

Since $A \backslash B\left(x_{0}, \gamma R\right) \subset \bigcup_{n \in \mathbb{N}} U_{n}$, we know, by Proposition 1.3, that

$$
\sum_{n \in \mathbb{N}} R_{1}^{U_{n}}=\infty
$$

By [2, VI.1.7], there exist open sets $V_{n}$ such that $\bar{V}_{n}$ is compact in $U_{n}$ and

$$
R_{1}^{U_{n}}\left(x_{0}\right) \leq R_{1}^{V_{n}}\left(x_{0}\right)+2^{-n}, \quad n \in \mathbb{N} .
$$

Then, by (3.2),

$$
\sum_{n \in \mathbb{N}} R_{1}^{V_{n}}\left(x_{0}\right)=\infty
$$

Let $n_{0} \in \mathbb{N}$ such that $\gamma^{n_{0}} R / 2>R_{0}$. For $n \geq n_{0}$, let $\nu_{n}:=\mu_{V_{n}}$, that is, $G \nu_{n}=R_{1}^{V_{n}}$. Since $\nu_{n}$ is supported by the set $\bar{V}_{n}$, which does not intersect $B\left(x_{0}, \gamma^{n} R / 2\right)$, and

$$
G\left(x_{0}, \cdot\right) \leq c g\left(\rho\left(x_{0}, \cdot\right)\right) \leq c g\left(\rho\left(\gamma^{n} R / 2\right)\right) \leq c c_{D} g\left(\gamma^{n} R\right) \quad \text { on } X \backslash B\left(x_{0}, \gamma^{n} R / 2\right) \text {, }
$$

we see that $R_{1}^{V_{n}}\left(x_{0}\right)=\int G\left(x_{0}, y\right) d \nu_{n}(y) \leq c c_{D} g\left(\gamma^{n} R\right)\left\|\nu_{n}\right\| \leq c c_{D} g\left(\gamma^{n} R\right) \operatorname{cap} U_{n}$. Therefore

$$
\infty=\sum_{n \geq n_{0}} R_{1}^{V_{n}}\left(x_{0}\right) \leq \sum_{n \geq n_{0}} c c_{D} g\left(\gamma^{n} R\right) \operatorname{cap} U_{n}
$$

Since $g\left(\gamma^{n} R\right) \leq g(R)<\infty$, for every $n \in \mathbb{N}$, we finally conclude from (3.1) that $\sum_{n \in \mathbb{N}} g\left(\gamma^{n} R\right) \operatorname{cap}^{*} A_{n}=\infty$.

Knowing that positive harmonic functions on $X$ are constant, by Corollary 2.2, a set $A$ in $X$ is avoidable if and only if it is minimally thin at infinity (see [11, Proposition 2.3]). Therefore it suffices to modify the proofs for [2, V.4.15 and V.4.17] (characterizing, in the setting of Riesz potentials, thinness of a set $A$ at a point). In the context of Lévy processes, this has already been noted (see, for example, 14, Proposition 7.3 and Corollary 7.4]). In our situation, the zero-one law will yield a straight forward modification.

THEOREM 3.2. Let $A \subset X, x_{0} \in X, s_{n} \in(0, \infty)$ and $\delta \in(0,1)$ with $s_{n} \leq \delta s_{n+1}$ for every $n \in \mathbb{N}$. Then the following hold for the sets $A_{n}:=A \cap S\left(x_{0}, s_{n}, s_{n+1}\right)$ :

(i) If $A$ is unavoidable, then $\sum_{n \in \mathbb{N}} R_{1}^{A_{n}}=\infty$ on $X$.

(ii) If $A$ is avoidable, then $\sum_{n \in \mathbb{N}} R_{1}^{A_{n}}<\infty$ on $X$.

Proof. (i) Proposition [1.3. (b).

(ii) By Proposition 1.1, there exists a point $x_{1} \in X$ such that $R_{1}^{A}\left(x_{1}\right)<\left(c^{2} c_{D}\right)^{-1}$. By [2, VI.1.2], there exists an open neighborhood $V$ of $A$ such that

$$
a:=c^{2} c_{D} R_{1}^{V}\left(x_{1}\right)<1 \text {. }
$$


The Liouville property implies that $R_{1}^{V}$ is a potential (see [11, Proposition 2.3]). Let

$$
V_{n}:=V \cap S\left(x_{1}, s_{n}, s_{n+3}\right), \quad n \in \mathbb{N} .
$$

Since $A_{n} \subset V_{n-1}$ if $n$ is sufficiently large, it suffices to show that

$$
\sum_{n \in \mathbb{N}} R_{1}^{V_{n}}<\infty
$$

To prove (3.4) let $k \in \mathbb{N}$ such that $1-\delta^{k-3}>1 / 2$. We fix $1 \leq i \leq k$ and define

$$
U_{n}:=V_{i+n k}, \quad n \in \mathbb{N} .
$$

It clearly suffices to show that $\sum_{n \in \mathbb{N}} R_{1}^{U_{n}}<\infty$. Let $U:=\bigcup_{n \in \mathbb{N}} U_{n}$. Since $R_{1}^{U} \leq R_{1}^{V}$, $R_{1}^{U}$ is a potential as well, $R_{1}^{U}=G \mu_{U}$. For $n \in \mathbb{N}$, let

$$
\mu_{n}:=1_{\bar{U}_{n}} \mu_{U}
$$

so that $\sum_{n \in \mathbb{N}} G \mu_{n}=G \mu_{U}=R_{1}^{U} \leq 1$.

Let $n_{0} \in \mathbb{N}$ such that $s_{n_{0}}>R_{0}$. For the moment, let us fix $n \geq n_{0}$, consider $m \in \mathbb{N}, m \neq n, y \in U_{n}$, and $z \in \bar{U}_{m}$. If $m<n$, then $\rho\left(y_{1}, z\right) \leq \delta^{k-3} \rho\left(x_{1}, y\right)$. If $m>n$, then $\rho\left(x_{1}, y\right) \leq \delta^{k-3} \rho\left(x_{1}, z\right)$. In both cases,

$$
\rho(y, z) \geq\left(1-\delta^{k-3}\right) \rho\left(x_{1}, z\right) \geq \rho\left(x_{1}, z\right) / 2 .
$$

Defining $\mu_{n}^{\prime}:=\mu_{U}-\mu_{n}=\sum_{m \neq n} \mu_{m}$ we hence obtain that, for every $y \in U_{n}$,

$$
G \mu_{n}^{\prime}(y) \leq c \int g(\rho(y, z)) d \mu_{n}^{\prime}(z) \leq c c_{D} \int g\left(\rho\left(x_{1}, z\right)\right) d \mu_{n}^{\prime}(z) \leq c^{2} c_{D} G \mu_{n}^{\prime}\left(x_{1}\right) \leq a .
$$

Since $G \mu_{n}+G \mu_{n}^{\prime}=G \mu_{U}=R_{1}^{U}$ and $R_{1}^{U}=1$ on $U$, we therefore conclude that $1-a \leq G \mu_{n}$ on $U_{n}$, and hence

$$
(1-a) R_{1}^{U_{n}} \leq G \mu_{n}
$$

Thus $\sum_{n \geq n_{0}} R_{1}^{U_{n}} \leq(1-a)^{-1} \sum_{n \geq n_{0}} G \mu_{n} \leq(1-a)^{-1} G \mu_{U} \leq(1-a)^{-1}$ completing the proof.

Proof of Theorem 1.12. Let $A \subset X, x_{0} \in X, R>0$ and $\gamma>1$.

If $A$ is unavoidable, then (1.12) holds, by Proposition 3.1

So let us assume that $A$ is avoidable. By [2, VI.1.5], there exists an open neighborhood $U$ of $A$ which is avoidable. For every $n \in \mathbb{N}$,

$$
U_{n}:=U \cap S\left(x_{0}, \gamma^{n-1} R, \gamma^{n+1} R\right)
$$

is an open neighborhood of $A \cap S\left(x_{0}, \gamma^{n} R, \gamma^{n+1} R\right)$. By Proposition 3.2,

$$
\sum_{n \in \mathbb{N}} R_{1}^{U_{n}}\left(x_{0}\right)<\infty
$$

By Lemma 1.6, there exist open sets $V_{n}$ in $U_{n}$ such that $\bar{V}_{n}$ is compact in $U_{n}$ and

$$
\operatorname{cap} U_{n} \leq c^{2}\left\|\mu_{V_{n}}\right\|+2^{-n}, \quad n \in \mathbb{N} .
$$


Let $k \in \mathbb{N}$ such that $\gamma \leq 2^{k}$, and let $n \in \mathbb{N}$ such that $\gamma^{n} R>R_{0}$. Then $g\left(\gamma^{n} R\right) \leq$ $c_{D}^{k} g\left(\gamma^{n+1} R\right) \leq c_{D}^{k} g\left(\rho\left(x_{0}, \cdot\right)\right)$ on $\bar{V}_{n}$, and hence

$$
g\left(\gamma^{n} R\right)\left\|\mu_{V_{n}}\right\| \leq c_{D}^{k} \int g\left(\rho\left(x_{0}, y\right)\right) d \mu_{V_{n}}(y) \leq c c_{D}^{k} G \mu_{V_{n}}\left(x_{0}\right)
$$

Since $G \mu_{V_{n}}=R_{1}^{V_{n}} \leq R_{1}^{U_{n}}$ and $g\left(\gamma^{n} R\right) \leq g(R), n \in \mathbb{N}$, we conclude that

$$
\sum_{n \in \mathbb{N}} g\left(\gamma^{n} R\right) \operatorname{cap} U_{n}<\infty
$$

Thus $\sum_{n \in \mathbb{N}} g\left(\gamma^{n} R\right) \operatorname{cap}^{*}\left(A \cap S\left(x_{0}, \gamma^{n} R, \gamma^{n+1} R\right)\right)<\infty$.

\section{Application to collections of balls having the separation property}

COROLLARY 4.1. Let $B\left(z, r_{z}\right), z \in Z \subset X, r_{z}>0$, be balls in $X$ such that their union $A$ is unavoidable. Then, for every $x_{0} \in X$,

$$
\sum_{z \in Z} g\left(\rho\left(x_{0}, z\right)\right) / g\left(r_{z}\right)=\infty
$$

Proof. Propositions 1.3 and 1.7.

The next simple result on comparison of potentials (cf. [7]) will be sufficient for us (see the proof of [11, Theorem 5.3] for a much more delicate version; cf. also the proof of [1, Theorem 3]).

LEMMA 4.2. Let $Z \subset \mathbb{R}^{d}$ be finite and $r_{z}>R_{0}, z \in Z$, such that, for $z \neq z^{\prime}$, $B\left(z, r_{z}\right) \cap B\left(z^{\prime}, 3 r_{z^{\prime}}\right)=\emptyset$. Let $w \in \mathcal{W} \cap \mathcal{C}(X)$ and, for every $z \in Z$, let $\mu_{z}, \nu_{z}$ be measures on $B\left(z, r_{z}\right)$ such that $G \mu_{z} \leq w$, and $\left\|\mu_{z}\right\| \leq\left\|\nu_{z}\right\|$. Then $\mu:=\sum_{z \in Z} \mu_{z}$ and $\nu:=\sum_{z \in Z} \nu_{z}$ satisfy

$$
G \mu \leq w+c^{2} c_{D} G \nu
$$

Proof. Let $z, z^{\prime} \in Z, z^{\prime} \neq z$, and $x \in B\left(z, r_{z}\right)$. For all $y, y^{\prime} \in B\left(z^{\prime}, r_{z^{\prime}}\right), \rho\left(y, y^{\prime}\right)<$ $2 r_{z^{\prime}}<\rho(x, y)$, hence $\rho\left(x, y^{\prime}\right) \leq 2 \rho(x, y), g(\rho(x, y)) \leq c_{D} g(2 \rho(x, y)) \leq c_{D} g\left(\rho\left(x, y^{\prime}\right)\right)$, and $G(x, y) \leq c^{2} c_{D} G\left(x, y^{\prime}\right)$. By integration, $G \mu_{z^{\prime}}(x) \leq c^{2} c_{D} G \nu_{z^{\prime}}(x)$. Therefore

$$
G \mu(x)=G \mu_{z}(x)+\sum_{z^{\prime} \in Z, z^{\prime} \neq z} G \mu_{z^{\prime}}(x) \leq w(x)+c^{2} c_{D} G \nu(x) .
$$

Thus $G \mu \leq w+c^{2} c_{D} G \nu$ on the union $A$ of the balls $B\left(z, r_{z}\right), z \in Z$. By the minimum principle (see [2, III.6.6]), the proof is finished.

LEMMA 4.3. Let $x_{0} \in X, R>2 R_{0}$ and $B:=B\left(x_{0}, R\right)$. Suppose that there exist $C \geq 1$ and a probability measure $\lambda$ on $B$ such that $G \lambda \leq C g(R)$. Let $Z$ be a finite subset of $B\left(x_{0}, R / 2\right)$ and $R_{0}<r_{z} \leq R / 2, z \in Z$, such that the balls $B\left(z, 3 r_{z}\right)$ are pairwise disjoint and, for some $\varepsilon \in(0,1)$,

$$
g\left(r_{z}\right) \lambda\left(B\left(z, \rho\left(z, z^{\prime}\right) / 4\right)\right) \geq \varepsilon g(R), \quad \text { whenever } z \neq z^{\prime} .
$$


Then the union $A$ of the balls $B\left(z, r_{z}\right), z \in Z$, satisfies

$$
\text { cap } A \geq \varepsilon\left(2 c^{3} c_{D} C\right)^{-1} \sum_{z \in Z} \operatorname{cap} B\left(z, r_{z}\right) .
$$

Proof. It clearly suffices to consider the case, where $Z$ contains more than one point. Then, for $z \in Z$,

$$
\tilde{r}_{z}:=\max \left\{r_{z}, \operatorname{dist}(z, Z \backslash\{z\}) / 4\right\} \leq R / 2,
$$

hence $B\left(z, \tilde{r}_{z}\right) \subset B$ and $\lambda\left(B\left(z, \tilde{r}_{z}\right)\right)>0$, by (4.2). Further, $B\left(z, \tilde{r}_{z}\right) \cap B\left(z^{\prime}, 3 \tilde{r}_{z^{\prime}}\right)=\emptyset$, whenever $z \neq z^{\prime}$,

For $z \in Z$, let $\mu_{z} \in \mathcal{M}(X)$ with $\mu_{z}\left(X \backslash B\left(z, r_{z}\right)\right)=0$ and $G \mu_{z} \leq 1$, and let

$$
\alpha_{z}:=\left\|\mu_{z}\right\| / \lambda\left(B\left(z, \tilde{r}_{z}\right)\right), \quad \nu_{z}:=\alpha_{z} 1_{B\left(z, \tilde{r}_{z}\right)} \lambda .
$$

Then $\left\|\nu_{z}\right\|=\left\|\mu_{z}\right\|$ and, by Proposition 1.7. (4.3), and (4.2),

$$
\alpha_{z} \leq c g\left(r_{z}\right)^{-1} / \lambda\left(B\left(z, \tilde{r}_{z}\right)\right) \leq c(\varepsilon g(R))^{-1} .
$$

Since the balls $B\left(z, \tilde{r}_{z}\right), z \in Z$, are pairwise disjoint subsets of $B$, the measure $\nu:=\sum_{z \in Z} \nu_{z}$ satisfies

$$
G \nu \leq c(\varepsilon g(R))^{-1} G \lambda \leq c C \varepsilon^{-1} .
$$

Let $\mu:=\sum_{z \in Z} \mu_{z}$. By Lemma 4.2, $G \mu \leq 1+c^{2} c_{D} G \nu$. Thus $G \mu \leq 2 c^{3} c_{D} C \varepsilon^{-1}$. Since $\mu(X \backslash A)=0$, we see that cap $A \geq \varepsilon\left(2 c^{3} c_{D} C\right)^{-1} \sum_{z \in Z}\left\|\mu_{z}\right\|$ completing the proof.

In addition to the Assumptions 1.4 and 1.8, we suppose the following.

ASSUMPTION 4.4. We have a measure $\lambda \in \mathcal{M}(X)$ and a constant $c_{0}>0$ such that $\operatorname{supp}(\lambda)=X$ and, for all $x \in X$ and $r>R_{0}$,

$$
G \lambda_{B(x, r)} \leq c_{0} g(r)
$$

(where, as before, $\lambda_{B}:=\lambda(B)^{-1} 1_{B} \lambda$ for every ball $B$ ).

We already observed that then, for all $x \in X$ and $r>R_{0}$,

$$
\operatorname{cap} B(x, r) \geq c_{0}^{-1} g(r)^{-1}
$$

(see Proposition 1.7 for the corresponding upper estimate). By Proposition 1.10, such a lower estimate for the capacity of balls is equivalent to having inequalities $R_{1}^{B(x, r)} \geq C^{-1} G(\cdot, x) / g(r)$ on $X \backslash B(x, r)$ (which in turn, by the minimum principle, hold trivially if $(X, \mathcal{W})$ is a harmonic space, that is, if $\mathfrak{X}$ has no jumps).

Let us say that a family of pairwise disjoint balls $B\left(z, r_{z}\right), z \in Z \subset X, r_{z}>2 R_{0}$, has the separation property with respect to $\lambda$, if $Z$ is locally finite and, for some point $x_{0} \in X \backslash Z$,

$$
\inf _{z, z^{\prime} \in Z, z \neq z^{\prime}} \frac{\lambda\left(B\left(z, \rho\left(z, z^{\prime}\right) / 4\right)\right)}{\lambda\left(B\left(x_{0}, 4 \rho\left(x_{0}, z\right)\right)\right)} \cdot \frac{g\left(r_{z}\right)}{g\left(\rho\left(x_{0}, z\right)\right)}>0 .
$$


REMARK 4.5. If, for example, $\lambda$ is Lebesgue measure on $X=\mathbb{R}^{d}, x_{0}=0$, $\rho(x, y)=|x-y|$, and $g(r)=r^{\alpha-d}$, then (4.6) means that

$$
\inf _{z, z^{\prime} \in Z, z \neq z^{\prime}} \frac{\left|z-z^{\prime}\right|^{d}}{|z|^{\alpha} r_{z}^{d-\alpha}}>0,
$$

which, in the classical case $\alpha=2$, is the separation property in [3, Theorem 6].

THEOREM 4.6. Let $A$ be an avoidable union of pairwise disjoint balls $B\left(z, r_{z}\right)$, $z \in Z \subset X$, having the separation property with respect to $\lambda$. Then

$$
\sum_{z \in Z} g\left(\rho\left(x_{0}, z\right)\right) \operatorname{cap} B\left(z, r_{z}\right)<\infty .
$$

Proof. We may suppose that $\rho\left(x_{0}, z\right)>4 R_{0}$, for every $z \in Z$ (we simply omit finitely many points from $Z$ ). Moreover, we may assume without loss of generality that

$$
r_{z} \leq \rho\left(x_{0}, z\right) / 2, \quad \text { for every } z \in Z .
$$

Indeed, replacing $r_{z}$ by $r_{z}^{\prime}:=\min \left\{r_{z}, \rho\left(x_{0}, z\right) / 2\right\}$ our assumptions are preserved. Suppose we have shown that $\sum_{z \in Z} g\left(\rho\left(x_{0}, z\right)\right) / g\left(r_{z}^{\prime}\right)<\infty$. Since $g(r) / g(r / 2) \geq c_{D}^{-1}$ if $r>2 R_{0}$, the set $Z^{\prime}$ of all points $z \in Z$ such that $r_{z}^{\prime}=\rho\left(x_{0}, z\right) / 2$ is finite, and therefore $\sum_{z \in Z^{\prime}} g\left(\rho\left(x_{0}, z\right)\right) / g\left(r_{z}\right)<\infty$. So we may assume without loss of generality that $r_{z}^{\prime}=r_{z}$, for all $z \in Z$, that is, (4.7) holds.

Further, we may assume that the balls $B\left(z, 4 r_{z}\right)$ are pairwise disjoint. Indeed, since cap $B\left(z, r_{z}\right) \approx g\left(r_{z}\right)^{-1}$ and $g(r) \leq g(r / 4) \leq c_{D}^{2} g(r), r>2 R_{0}$, a replacement of $r_{z}$ by $r_{z} / 4$ does neither affect (4.6) nor the convergence or divergence of $\sum_{z \in Z} g\left(\rho\left(x_{0}, z\right)\right) \operatorname{cap} B\left(z, r_{z}\right)$, and the new, smaller union is, of course, avoidable.

By (4.6), there exists $\varepsilon \in(0,1)$ such that, for $z, z^{\prime} \in Z, z \neq z^{\prime}$,

$$
g\left(r_{z}\right) \lambda\left(B\left(z, \rho\left(z, z^{\prime}\right) / 4\right)\right) \geq \varepsilon g\left(\rho\left(x_{0}, z\right)\right) \lambda\left(B\left(x_{0}, 4 \rho\left(x_{0}, z\right)\right)\right) .
$$

Let $R>R_{0}$. For $n \in \mathbb{N}$, let

$$
Z_{n}:=Z \cap S\left(x_{0}, 2 \cdot 8^{n} R, 4 \cdot 8^{n} R\right) \quad \text { and } \quad A_{n}:=\bigcup_{z \in Z_{n}} B\left(z, r_{z}\right) .
$$

Then $A_{n} \subset A \cap S\left(x_{0}, 8^{n} R, 8^{n+1} R\right)$. Moreover, for every $z \in Z_{n}, \rho\left(x_{0}, z\right) \geq 8^{n} R$, and hence $g\left(\rho\left(x_{0}, z\right)\right) \leq g\left(8^{n} R\right)$. Therefore, by Lemma 4.3 and Theorem 1.12,

$$
\begin{aligned}
& \sum_{n \in \mathbb{N}} \sum_{z \in Z_{n}} g\left(\rho\left(x_{0}, z\right)\right) \operatorname{cap} B\left(z, r_{z}\right) \\
& \leq 2 c^{3} c_{D} c_{0} \varepsilon^{-1} \sum_{n \in \mathbb{N}} g\left(8^{n} R\right) \operatorname{cap}^{*}\left(A \cap S\left(x_{0}, 8^{n} R, 8^{n+1} R\right)\right)<\infty .
\end{aligned}
$$

Applying this estimate as well to $2 R$ and $4 R$ in place of $R$ we obtain that

$$
\sum_{z \in Z} g\left(\rho\left(x_{0}, z\right)\right) \operatorname{cap} B\left(z, r_{z}\right)<\infty \text {. }
$$


COROLLARY 4.7. For every union $A$ of balls $B\left(z, r_{z}\right), z \in Z \subset X$, having the separation property with respect to $\lambda$, the following statements are equivalent.

(1) The set $A$ is unavoidable.

(2) $\sum_{z \in Z} g\left(\rho\left(x_{0}, z\right)\right) \operatorname{cap} B\left(z, r_{z}\right)=\infty$.

(3) $\sum_{z \in Z} g\left(\rho\left(x_{0}, z\right)\right) / g\left(r_{z}\right)=\infty$.

Proof. Corollary 4.1 and Theorem 4.6 using cap $B\left(z, r_{z}\right) \approx g\left(r_{z}\right)^{-1}, z \in Z$.

\section{Application to regularly located balls}

In this section we suppose as before that the Assumptions 1.4, 1.8, and 4.4 are satisfied. Moreover, let us assume that we have a distinguished point $x_{0} \in X$ such that the measure $\lambda$ has the following additional properties:

(i) For all $x, y \in X$ and $r>R_{0}$,

$$
\lambda(B(y, r)) \leq c_{0} \lambda(B(x, r)) .
$$

(ii) There exist $C_{D} \in(1, \infty), \kappa \in(0,1)$ such that, for all $r>R_{0}$,

$$
\lambda\left(B\left(x_{0}, 2 r\right)\right) \leq C_{D} \lambda\left(S\left(x_{0}, \kappa r, r\right)\right) .
$$

Let $A$ be a union of balls $B\left(z, r_{z}\right), z \in Z$, which is regularly located (see 1.14). We first prove the following proposition.

PROPOSITION 5.1. Suppose that $\limsup _{r \rightarrow \infty} \lambda\left(B\left(x_{0}, r\right)\right) g(r) / g(\phi(r))>0$. Then $A$ is unavoidable.

Proof. We may assume without loss of generality that the radius $R$ in (1.14) satisfies $R>\max \left\{1, \phi(1), R_{0}\right\}$. We define

$$
a:=\left(c c_{0}^{2} C_{D}^{5} \lambda\left(B\left(x_{0}, R\right)\right)^{-1} \quad \text { and } \quad b:=c c_{0}^{2} \lambda\left(B\left(x_{0}, R\right)\right)^{-1} .\right.
$$

Let $0<\beta<\lim \sup _{r \rightarrow \infty} \lambda\left(B\left(x_{0}, r\right)\right) g(r) / g(\phi(r))$.

We now fix $x \in X$ and choose $r>\kappa^{-1}\left(4 R+2 \rho\left(x_{0}, x\right)\right)$ such that $r>\phi(r)$ and

$$
\gamma:=\lambda\left(B\left(x_{0}, r\right)\right) g(r) / g(\phi(r))>\beta
$$

Let

$$
S:=S\left(x_{0}, \kappa r / 2, r / 2\right), \quad B:=B\left(x_{0}, r\right) \quad \text { and } \quad r_{0}:=\phi(r)
$$

so that

$$
\gamma=\lambda(B) g(r) / g\left(r_{0}\right)
$$


There are finitely many points $y_{1}, \ldots, y_{m} \in \bar{S}$ such that $B\left(y_{1}, 3 R\right), \ldots, B\left(y_{m}, 3 R\right)$ are pairwise disjoint and $\bar{S}$ is covered by $B\left(y_{1}, 9 R\right), \ldots, B\left(y_{m}, 9 R\right)$. We may choose $z_{j} \in Z \cap B\left(y_{j}, R\right), 1 \leq j \leq m$. Then $\rho\left(z_{i}, z_{j}\right) \geq \rho\left(y_{i}, y_{j}\right)-2 R \geq 4 R$, and hence

$$
B\left(z_{i}, R\right) \cap B\left(z_{j}, 3 R\right)=\emptyset
$$

for all $i, j \in\{1, \ldots, m\}$ with $i \neq j$. Moreover,

$$
\lambda(B) \leq C_{D} \lambda(S) \leq C_{D} \sum_{j=1}^{m} \lambda\left(B\left(y_{j}, 9 R\right)\right) \leq m C_{D}^{5} c_{0} \lambda\left(B\left(x_{0}, R\right)\right) .
$$

Let $1 \leq j \leq m$. Clearly,

$$
r>r-R>r / 2+R \geq \rho\left(x_{0}, z_{j}\right) \geq \kappa r / 2-R \geq R \geq 1 .
$$

Therefore $B\left(z_{j}, R\right) \subset B$ and $r_{0}=\phi(r) \leq \phi\left(\rho\left(x_{0}, z_{j}\right)\right)<r_{z_{j}}$, hence $\bar{B}\left(z_{j}, r_{0}\right) \subset A$. Moreover, $r_{0} \leq \phi(1) \leq R$ and $r_{0}+\rho\left(x, z_{j}\right) \leq R+\rho\left(x_{0}, x\right)+r / 2+R \leq r$. So $g\left(\rho\left(x, z_{j}\right)+r_{0}\right) \geq g(r)$ and, by (1.10) and (4.5),

$$
R_{1}^{B\left(z_{j}, r_{0}\right)}(x) \geq\left(c c_{0}\right)^{-1} g\left(\rho\left(x, z_{j}\right)+r_{0}\right) / g\left(r_{0}\right) \geq\left(c c_{0}\right)^{-1} g(r) / g\left(r_{0}\right) .
$$

Let $\mu_{j}$ be the equilibrium measure for $B\left(z_{j}, r_{0}\right), 1 \leq j \leq m$. We define

$$
p:=\sum_{j=1}^{m} R_{1}^{B\left(z_{j}, r_{0}\right)}=\sum_{j=1}^{m} G \mu_{j} .
$$

Then, by (5.6) and (5.5),

$$
p(x) \geq m\left(c c_{0}\right)^{-1} g(r) / g\left(r_{0}\right) \geq a \gamma .
$$

Finally, let $\nu:=\sum_{j=1}^{m} \nu_{j}$, where

$$
\nu_{j}:=c g\left(r_{0}\right)^{-1} \lambda_{B\left(z_{j}, R\right)} \leq c c_{0} g\left(r_{0}\right)^{-1} \frac{\lambda(B)}{\lambda\left(B\left(x_{0}, R\right)\right)} 1_{B\left(z_{j}, R\right)} \lambda_{B} .
$$

Since $B\left(z_{1}, R\right), \ldots, B\left(z_{j}, R\right)$ are pairwise disjoint subsets of $B$ and $G \lambda_{B} \leq c_{0} g(r)$,

$$
G \nu \leq c c_{0} g\left(r_{0}\right)^{-1} \frac{\lambda(B)}{\lambda\left(B\left(x_{0}, R\right)\right)} G \lambda_{B} \leq c c_{0}^{2} \frac{\lambda(B)}{\lambda\left(B\left(x_{0}, R\right)\right)} g(r) / g\left(r_{0}\right)=b \gamma .
$$

By Proposition 1.7, $\left\|\mu_{j}\right\| \leq c g\left(r_{0}\right)^{-1}, 1 \leq j \leq m$. Thus, by (5.4) and Lemma 4.2,

$$
p \leq 1+c^{2} c_{D} G \nu \leq 1+c^{2} c_{D} b \gamma
$$

Since $\mu$ is supported by the compact $\bar{B}\left(z_{1}, r_{0}\right) \cup \cdots \cup \bar{B}\left(z_{m}, r_{0}\right)$ in $A$, this implies that

$$
R_{1}^{A} \geq\left(1+c^{2} c_{D} b \gamma\right)^{-1} p,
$$

by the minimum principle (see [2, III.6.6]). In particular,

$$
R_{1}^{A}(x) \geq \frac{a \gamma}{1+c^{2} c_{D} b \gamma}=\frac{a}{\gamma^{-1}+c^{2} c_{D} b}>\frac{a}{\beta^{-1}+c^{2} c_{D} b},
$$

by (5.7) and (5.3). Thus $A$ is unavoidable, by Proposition 1.1. 
Proof of Corollary 1.15. If $A$ is unavoidable, then $\sum_{z \in Z} g\left(\rho\left(x_{0}, z\right)\right) / g\left(r_{z}\right)=\infty$, by Corollary 4.1 .

To prove the converse, suppose that $\sum_{z \in Z} g\left(\rho\left(x_{0}, z\right)\right) / g\left(r_{z}\right)=\infty$. By Proposition 5.1 and (5.2), it suffices to consider the case

$$
\lim _{r \rightarrow \infty} \lambda\left(B\left(x_{0}, 4 r\right)\right) g(r) / g(\phi(r))=0 .
$$

Then $\left.\inf _{r>0} g(\phi(r))\left(\lambda\left(B\left(x_{0}, 4 r\right)\right) g(r)\right)\right)^{-1}>0$. By (1.15),$g\left(\phi\left(r_{z}\right)\right) \approx g\left(\rho\left(x_{0}, z\right)\right)$, $z \in Z$. Moreover,

$$
\lambda\left(B\left(z, \rho\left(z, z^{\prime}\right) / 4\right)\right) \geq \inf _{x \in X} \lambda(B(x, \varepsilon / 4))>0
$$

whenever, $z, z^{\prime} \in Z, z \neq z^{\prime}$. So the balls $B\left(z, r_{z}\right), z \in Z$, have the separation property, and $A$ is unavoidable, by Corollary 4.7.

\section{Appendix}

The following equivalences are of independent interest and may be useful in applications.

PROPOSITION 6.1. Let $X$ be an arbitrary set and $G: X \times X \rightarrow[0, \infty]$ such that $G=\infty$ on the diagonal and $0<G<\infty$ outside the diagonal. Then the following properties are equivalent:

(i) $G$ has the triangle property: There exists $C \geq 1$ such that

$$
\min \{G(x, z), G(y, z)\} \leq C G(x, y), \quad x, y, z \in X
$$

(ii) There exists a metric $\rho$ on $X$ and $\gamma>0$ such that $G \approx \rho^{-\gamma}$.

(iii) There exist a metric $\rho$ on $X$, a decreasing function $g:[0, \infty) \rightarrow(0, \infty], c_{D} \geq 1$, and $\eta_{0}, \alpha_{0} \in(0,1)$ such that $G \approx g \circ \rho$ and, for every $r>0$,

$$
g(r / 2) \leq c_{D} g(r) \quad \text { and } \quad g(r) \leq \eta_{0} g\left(\alpha_{0} r\right) .
$$

(iv) There exist a metric $\rho$ on $X$, a decreasing function $g:[0, \infty) \rightarrow(0, \infty]$, and $c_{D} \geq 1$ such that $G \approx g \circ \rho$ and $g(r / 2) \leq c_{D} g(r)$ for every $r>0$.

Proof. (i) $\Rightarrow$ (ii): Since $G=\infty$ on the diagonal, the triangle property implies that $G(y, x) \leq C G(x, y)$ and $\tilde{\rho}(x, y):=G(x, y)^{-1}+G(y, x)^{-1}, x, y \in X$, defines a quasimetric on $X$ which is equivalent to $G^{-1}$. By [12, Proposition 14.5] (see also [8, pp. 1209-1212] and [5]), there exists a metric $\rho$ for $X$ and $\gamma>0$ such that $\tilde{\rho} \approx \rho^{\gamma}$, and hence $G \approx \rho^{-\gamma}$.

$$
\begin{aligned}
& \text { (ii) } \Rightarrow \text { (iii): Trivial defining } g(r):=r^{-\gamma} \text {. } \\
& \text { (iii) } \Rightarrow \text { (iv): Trivial. }
\end{aligned}
$$


(iv) $\Rightarrow$ (i): Let $c>0$ such that $c^{-1} g \circ \rho \leq G \leq c g \circ \rho$ and let $x, y, z \in X$. Since $\rho(x, y) \leq \rho(x, z)+\rho(y, z)$, we know that $\rho(x, z) \geq \rho(x, y) / 2$ or $\rho(y, z) \geq \rho(x, y) / 2$. Therefore

$$
\begin{aligned}
\min \{G(x, z), G(y, z)\} & \leq c \min \{g(\rho(x, z)), g(\rho(y, z))\} \\
& \leq c g(\rho(x, y) / 2) \leq c c_{D} g(\rho(x, y)) \leq c^{2} c_{D} G(x, y) .
\end{aligned}
$$

REMARKS 6.2. 1 . If $X$ is a topological space and each function $G(\cdot, x), x \in X$, is lower semicontinuous and bounded at infinity, the $\rho$ is a metric for the topology of $X$.

2. If (iii) holds, then, for every $\eta \in(0,1)$, there exists $\alpha \in(0,1)$ such that $g(r) \leq \eta g(\alpha r)$. Indeed, it suffices to choose $k \in \mathbb{N}$ such that $\eta_{0}^{k}<\eta$ and to take $\alpha:=\alpha^{k}$.

\section{References}

[1] H. Aikawa and A.A. Borichev. Quasiadditivity and measure property of capacity and the tangential boundary behavior of harmonic functions. Trans. Amer. Math. Soc., 348:1013-1030, 1996.

[2] J. Bliedtner and W. Hansen. Potential Theory - An Analytic and Probabilistic Approach to Balayage. Universitext. Springer, Berlin, 1986.

[3] S.J. Gardiner and M. Ghergu. Champagne subregions of the unit ball with unavoidable bubbles. Ann. Acad. Sci. Fenn. Math., 35(1):321-329, 2010.

[4] T. Grzywny. On Harnack inequality and Hölder regularity for isotropic unimodal Lévy processes. Potential Anal. 41: 1-29, 2014.

[5] W. Hansen. Uniform boundary Harnack principle and generalized triangle property. J. Funct. Anal., 226: 452-484, 2005.

[6] W. Hansen. Three views on potential theory. A course at Charles University (Prague), Spring 2008. http://www.karlin.mff.cuni.cz/ hansen/lecture/ course-07012009.pdf.

[7] W. Hansen Unavoidable collections of balls for processes with isotropic unimodal Green function. In Festschrift Masatoshi Fukushima (eds. Z.-Q. Chen, N. Jacob, M. Takeda, T. Uemura), World Scientific Press, 2015.

[8] W. Hansen and I. Netuka. Convexity properties of harmonic measures. Adv. Math., 218(4):1181-1223, 2008.

[9] W. Hansen and I. Netuka. Champagne subdomains with unavoidable bubbles. Adv. Math. 244:106-116, 2013.

[10] W. Hansen and I. Netuka. Representation of potentials. Rev. Roumaine Math. Pures Appl., 59: 93-104, 2014. 
[11] W. Hansen and I. Netuka. Unavoidable sets and harmonic measures living on small sets. Proc. London Math. Soc. 109: 1601-1629, 2014.

[12] J. Heinonen. Lectures on analysis on metric spaces. Springer, New York, 2001.

[13] H. Maagli. Représentation intégrale des potentiels. In Séminaire de Théorie du Potentiel, Paris, No. 8, volume 1235 of Lecture Notes in Math., pages 114-119. Springer, Berlin, 1987.

[14] A. Mimica and Z. Vondraček. Unavoidable collections of balls for isotropic Lévy processes. Stochastic Process. Appl., 124(3):1303-1334, 2014.

Wolfhard Hansen, Fakultät für Mathematik, Universität Bielefeld, 33501 Bielefeld, Germany, e-mail: hansen@math.uni-bielefeld.de 